\title{
BOUNDEDNESS OF SOLUTIONS TO LINEAR DIFFERENTIAL EQUATIONS
}

\author{
BY ANTHONY J. SCHAEFFER
}

Communicated by L. Cesari, January 2, 1968

In the case of a linear constant coefficient differential equation, $\dot{x}=A x$, where $x$ is a (complex) $n$-vector and $A$ is a (complex) $n \times n$ matrix, it is well known when all solutions are bounded; namely, if all eigenvalues of $A$ are purely imaginary and all elementary divisions of $A$ are simple. This condition is equivalent to the Jordan normal form, $J$, of $A$ being (Hermitian) skew symmetric. That is if $J=P A P^{-1}$, then

$$
J+J^{*}=P A P^{-1}+P^{*-1} A^{*} P^{*}=0,
$$

where $M^{*}$ denotes the adjoint or complex conjugated transpose of $M$. Multiplying (1) on the left by $P^{*}$ and on the right by $P$ yields the equivalent condition that there exist a positive definite $Q=P^{*} P$ such that

$$
Q A+A^{*} Q=0 .
$$

In the time dependent case, it is shown here that a necessary and sufficient condition that all solutions of $\dot{x}=A(t) x$ be bounded is that there exist a $Q(t)$ that is uniformly bounded and uniformly positive definite and that satisfies

$$
Q(t) A(t)+A^{*}(t) Q(t)+Q(t)=0 .
$$
then

We will use the following notation. If $\xi$ and $\eta$ are complex $n$-vectors,

$$
\langle\xi, \eta\rangle=\sum_{i=1}^{n} \xi_{i} \bar{\eta}_{i}
$$

will denote the inner product of $\xi$ and $\eta$, and

$$
\|\xi\|=\langle\xi, \xi\rangle^{1 / 2}
$$

will denote the norm of $\xi$. Also $M^{*}$ will denote the adjoint matrix of a matrix $M$.

The author would like to thank James J. Hurt for several helpful discussions during this work.

THEOREM 1. Let $A(t)$ be an $n \times n$ matrix function defined and continuous on an open interval I. If there exists a continuously differentiable 
matrix function $Q(t)$ and real function $\gamma(t)$ and $\delta(t)$ defined on $I$ such that

$$
\begin{gathered}
Q(t) A(t)+A^{*} Q(t)+Q(t)=0, \\
\gamma(t)\|\xi\|^{2} \leqq\langle Q(t) \xi, \xi\rangle \leqq \delta(t)\|\xi\|^{2}, \\
0<\gamma(t) \leqq \delta(t)<\infty,
\end{gathered}
$$

for all $t$ in $I$; then all solutions of

$$
\dot{x}(t)=A(t) x(t)
$$

satisfy

$$
\gamma(t)\|x(t)\|^{2} \leqq \delta\left(t_{0}\right)\left\|x\left(t_{0}\right)\right\|^{2}
$$

for all $t, t_{0}$ in $I$.

Conversely, if there exists a real function $\alpha\left(t, t_{0}\right)$ defined in $I \times I$ such that

$$
\begin{aligned}
\|x(t)\|^{2} & \leqq \alpha\left(t, t_{0}\right)\left\|x\left(t_{0}\right)\right\|^{2}, \\
0 & <\alpha\left(t, t_{0}\right)<\infty
\end{aligned}
$$

for all $t, t_{0}$ in $I$ and any solution $x(t)$ of $(6)$, then there exist Hermitian symmetric matrices $Q_{r}(t)$ defined for $t$ in $I$ and satisfying (3) for each $\tau$ in I. Also

$$
\alpha^{-1}(t, \tau)\|\xi\|^{2} \leqq\left\langle Q_{\tau}(t) \xi, \xi\right\rangle \leqq \alpha(\tau, t)\|\xi\|^{2} .
$$

Proof. Suppose $Q, \gamma$, and $\delta$ exist satisfying (3), (4), and (5), and let $x(t)$ be any solution of (6). Then

$$
\begin{aligned}
(d / d t)\langle Q(t) & x(t), x(t)\rangle \\
& =\langle Q(t) x(t), x(t)\rangle+\langle Q(t) \dot{x}(t), x(t)\rangle+\langle Q(t) x(t), \dot{x}(t)\rangle \\
& =\left\langle\left[Q(t)+Q(t) A(t)+A^{*}(t) Q(t)\right] x(t), x(t)\right\rangle=0
\end{aligned}
$$

since (3) holds. Thus $\langle Q(t) x(t), x(t)\rangle$ is a constant, and

$$
\begin{aligned}
\gamma(t)\|x(t)\|^{2} & \leqq\langle Q(t) x(t), x(t)\rangle \\
& =\left\langle Q\left(t_{0}\right) x\left(t_{0}\right), x\left(t_{0}\right)\right\rangle \leqq \delta\left(t_{0}\right)\left\|x\left(t_{0}\right)\right\|^{2} .
\end{aligned}
$$

Now suppose (8) and (9) hold for all solutions of (6). Let $X\left(t, t_{0}\right)$ be the fundamental matrix of (6) such that $X\left(t_{0}, t_{0}\right)=I$, and define $Q_{\tau}(t)$ by

$$
Q_{\tau}(t)=X^{*}(\tau, t) X(\tau, t) .
$$

It is clear that $Q_{r}(t)$ is defined and differentiable for $t$ in $I$ and all $\tau$ in $I$. Also 


$$
\begin{aligned}
Q_{\tau}(t) & =\dot{X}^{*}(\tau, t) X(\tau, t)+X^{*}(\tau, t) X(\tau, t) \\
& =-A^{*}(t) X^{*}(\tau, t) X(\tau, t)-X^{*}(\tau, t) X(\tau, t) A(t) \\
& =-\left[Q_{\tau}(t) A(t)+A^{*}(t) Q_{\tau}(t)\right],
\end{aligned}
$$

which is equation (3). We use here the fact that

$$
(d / d t) X(\tau, t)=\dot{X}(\tau, t)=-X(\tau, t) A(t) .
$$

Clearly $Q_{\tau}(t)$ is Hermitian symmetric. Also

$$
\begin{aligned}
\left\langle Q_{\tau}(t) \xi, \xi\right\rangle & =\left\langle X^{*}(\tau, t) X(\tau, t) \xi, \xi\right\rangle \\
& =\|X(\tau, t) \xi\|^{2} \leqq \alpha(\tau, t)\|\xi\|^{2},
\end{aligned}
$$

by (8) since $X(\tau, t) \xi$ is a solution of (6) (as a function of $\tau$ ) and $X(t, t) \xi=\xi$. Similarly, we have

$$
\begin{aligned}
\|\xi\|^{2} & =\|X(t, \tau) X(\tau, t) \xi\|^{2} \\
& \leqq \alpha(t, \tau)\|X(\tau, t) \xi\|^{2}=\alpha(t, \tau)\left\langle Q_{\tau}(t) \xi, \xi\right\rangle . \quad \text { Q.E.D. }
\end{aligned}
$$

REMARK. We could have obtained the properties desired for $Q_{\tau}(t)$ by setting

$$
Q_{\tau}(t)=X^{*}(\tau, t) R X(\tau, t)
$$

where $R$ is any positive definite constant matrix. Observe that we have

$$
X^{*}\left(t, t_{0}\right) Q_{\tau}(t) X\left(t, t_{0}\right)=Q_{\tau}\left(t_{0}\right)
$$

which can be shown directly from (11) or from (3) and differentiation.

REMARK. If we make a change of variables in (6) by letting

$$
y(t)=P(t) x(t),
$$

then the differential equation for $y(t)$ becomes

$$
\dot{y}(t)=\left[P(t) A(t) P^{-1}(t)+P(t) P^{-1}(t)\right] y(t)=B(t) y(t) .
$$

If for some $P(t)$ the resulting $B(t)$ is skew symmetric, then $Q(t)$ $=P^{*}(t) P(t)$ will satisfy (3) as was shown for the constant coefficient case in the introduction. Further, if there is a differentiable $P(t)$ such that $Q(t)=P^{*}(t) P(t)$, then the corresponding $B(t)$ is skew symmetric.

Corollary. If in Theorem $1, I=(-\infty, \infty)$, then all solutions of (6) are bounded (in the sense of (8) with $\alpha$ a constant) if and only if there exists a $Q(t)$ satisfying (3), (4), and (5), but with $\gamma$ and $\delta$ constant.

THEOREM 2. Under the assumptions of Theorem 1 there is a constant matrix $Q$ satisfying (3), (4), and (5) (with constant $\alpha$ and $\delta$ ) if and only if the mean value 


$$
\widetilde{Q}=\lim _{T \rightarrow \infty} \frac{1}{2 T} \int_{-T}^{T} X^{*}(\tau, t) R X(\tau, t) d \tau
$$

exists (boundedly), is independent of $t$, and is nonsingular for some positive definite matrix $R$.

Proof. If $Q$ exists and is constant, then $X^{*}(\tau, t) Q X(\tau, t)=Q$, and $\widetilde{Q}=Q$. If $\tilde{Q}$ exists and is independent of $t$, then

$$
\begin{aligned}
X^{*}\left(t, t_{0}\right) \tilde{Q} X\left(t, t_{0}\right) & =\lim _{T \rightarrow \infty} \frac{1}{2 T} \int_{-T}^{T} X^{*}\left(t, t_{0}\right) X^{*}(\tau, t) R X(\tau, t) X\left(t, t_{0}\right) d \tau \\
& =\lim _{T \rightarrow \infty} \frac{1}{2 T} \int_{-T}^{T} X^{*}\left(\tau, t_{0}\right) R X\left(\tau, t_{0}\right) d \tau=\tilde{Q} .
\end{aligned}
$$

This is equivalent to (3) by the remark made after Theorem 1. $\widetilde{Q}$ is clearly symmetric and nonnegative definite, but it is assumed nonsingular, so it must be positive definite. Q.E.D.

RemarK. If $A$ is constant, then $X\left(t, t_{0}\right)=X\left(t-t_{0}\right)=\exp A\left(t-t_{0}\right)$. Under our assumption that $\exp A \tau$ be bounded, it must be uniformly almost periodic. Thus

$$
\lim _{T \rightarrow \infty} \frac{1}{2 T} \int_{-T}^{T} X^{*}(\tau, t) X(\tau, t) d \tau
$$

exists and is independent of $t$. Thus there is a constant $Q$ as we know there must be. However, if $X\left(t, t_{0}\right)$ is periodic, there need not be a constant $Q$ satisfying (3) as can be seen by considering a scalar equation.

\section{UNIVERSITY OF IOWA}

